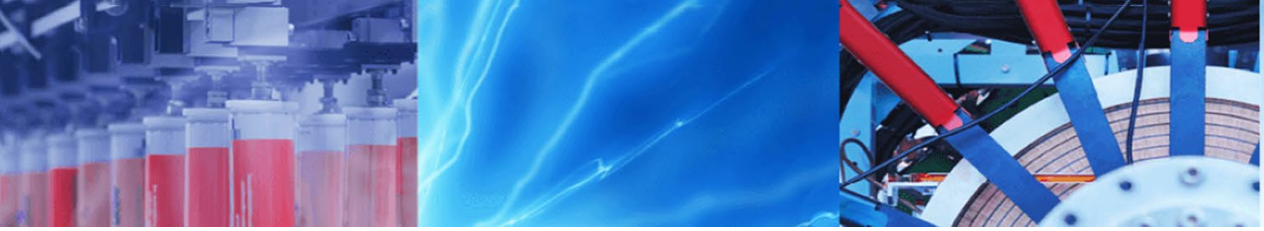

Research Article

\title{
A numerical and analytical study of the stress field generated by the contact between a rail and a wheel
}

\author{
André Oliveira Köhn ${ }^{1}$ (D) Fernando de Azevedo Silva ${ }^{1}$
}

Received: 30 January 2020 / Accepted: 10 June 2020 / Published online: 19 June 2020

(c) Springer Nature Switzerland AG 2020

\begin{abstract}
This article presents a study of the contact stresses between a rail and a wheel, in order to understand the mechanism related to this application. When two bodies, regardless of their forms, touch each other due to an external load, both suffer a slight deformation on the contact surface, creating a stress field in this area, and the complete comprehension of this phenomenon is fundamental to calculate critical stresses and to prevent possible failures. This article approaches this topic in two different manners: one analytical, using Hertz equations; and another numerical, using the FEM software Ansys. The results obtained from both approaches are compared, and the model can be validated. The results show that when these two cylindrical bodies are pressed against each other, a small rectangular area is created, and the highest normal stresses are found exactly where the two elements touch. It is seen that the maximum shear stress is found along the depth of the body and is the most critical for the appearance of cracks. The results from this work are in good alignment with reality and they can be used to predict possible failures and behaviors in real applications.
\end{abstract}

Keywords Hertz contact stresses · Deformation · Cylinder/plane contact · Wheel/rail contact · FEM model

\section{Introduction}

The study of contact stresses is a central topic in Solid Mechanics. When two surfaces, regardless of its geometric shapes, are pressed against each other due to an external load, a stress field is created in this contact area. This region is highly dependent on the applied load, mechanical properties of both elements and geometry from the bodies in contact [1]. Also, the maximum pressure value is much higher than the strength of the materials commonly found in engineering operations, reaching 1-4 GPa in typical applications like bearings [2, 3].

The first big contribution in this field was developed by Heinrich Hertz in 1882, in his paper On The Contact of Elastic Solids. In this study, Hertz proposed an analysis of the deformation caused by the contact between two elastic bodies with curved geometry [4].
This topic is important in many fields in Mechanics. In bearings, the contact between the rolling-elements and the inner and outer races creates a Hertzian stress zone that is defined by the model of the bearing: for example, a deep-groove ball bearing has a circumferential contact zone between the balls and the races $[5,6]$, while cylindrical roller bearings show a rectangular region between the surfaces in contact [3, 7-9]. Additionally, it is important to study the Hertzian stresses in gear teeth, subject of recent works like Hao and Shi [10], Hwang et al. [11], Patil et al. [12] and Gonzalez-Perez et al. [13], and in forming processes like calendering and lamination, where two or more cylinders are used to change the thickness of a material [1]. Recent articles like Nejad et al. [14], Xiao et al. [15] and Jaifu et al. [16] also discuss about how the Hertzian stresses are the critical causes to failures and cracks in between rail and wheels.

André Oliveira Köhn, andre.oliveira.kohn@gmail.com; Fernando de Azevedo Silva, fernando.azevedo@unesp.br| ${ }^{1}$ Guaratinguetá School of Engineering, UNESP, São Paulo State University, Av. Dr. Ariberto Pereira da Cunha 333, Pedregulho, Guaratinguetá, SP 12516-410, Brazil. 
The cyclic contact that happens between the rail and the wheel can cause a phenomenon called rolling contact fatigue (RCF). The load is applied cyclically, creating a triaxial compressive stress field [2,17], as well as the shear stress, which is the most critical for the appearance of cracks $[18,19]$. These stresses can be repeated millions of cycles, generating cracks that will be propagated until the total collapse of the element. These failures are expressed in the progressive loss of surface material, causing from small losses by pitting to severe and critical losses by spalling [1].

In this paper, the stress field between a rail and a wheel subjected to an external load was calculated and analyzed. An analytical approach was conducted using Hertz equations for contact stresses, and these results were compared to the data obtained from the FEM software Ansys. The behavior of the stresses on the contact plane was analyzed using graphics, as well as how the stresses vary in function of the depth. All calculations were based on the Hertz theory for a contact between two cylinders, and the equations were adapted to represent faithfully the case between a cylinder and a flat plate presented on the subject example.

This manuscript starts with a theoretical analysis of the problem, demonstrating all theory and equations used for the calculations, followed by a section where the elements used are discussed and explained. Then, the results are shown in two different sections: first, a section where the results obtained from the Hertz equations, and then a section with the numerical results obtained from the computer simulation. Henceforth, the results from both approaches are analyzed and discussed, with a section dedicated for the conclusions taken from this study.

\section{Theoretical analysis}

Ideally, when two cylinders and with radiuses $R_{1}$ and $R_{2}$, length $I$ and parallel axes are brought into contact with each other due to an external load, the contact region would be a single line parallel to their axes. However, as the stress is given by the division of the load by the contact area, this situation would generate an infinite stress, which is impossible to happen in reality. When the external load $F$ is applied, the contact region of both cylinders suffers a small deformation, creating a small rectangular area with length / and width $2 a$ as shown in Fig. 1 [3], and these dimensions are much smaller than the general dimensions of the cylinder $[4,20]$. The material in the region of contact is compressed equally in all directions, making it possible to endure higher stresses. Due to this characteristic, the strength generated in contact surfaces is higher than the usual material strength from other applications, reaching 4 GPa for steel [21].

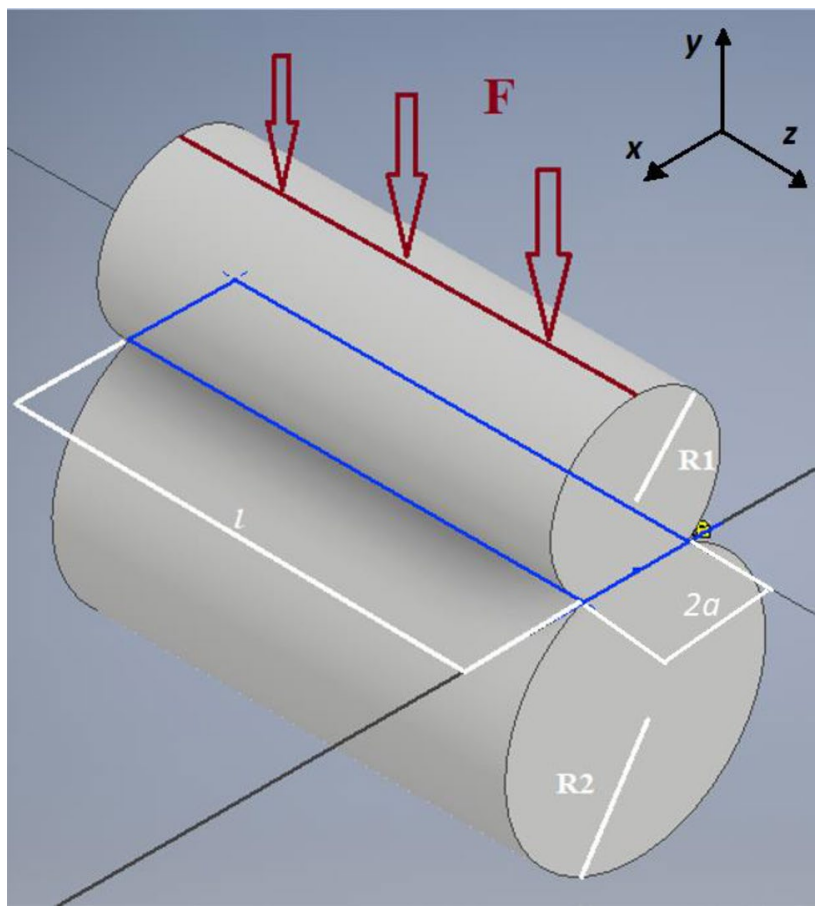

Fig. 1 Rectangular contact between two parallel cylinders

The Hertzian contact theory is based on four assumptions [4]:

1. Both bodies are considered as an elastic half-space;

2. The deformation area is significantly smaller if compared to the body dimensions $\left(a \ll R_{1} ; R_{2}\right)$;

3. The surfaces are frictionless;

4. The surfaces are continuous.

Knowing the dimensions of each cylinder and their mechanical properties (Young's modulus $E$ and Poisson ratio $u$ ), it is possible to obtain the stress field generated by the load $F$. Considering the plane where both solids touch as $x z$ (the $z$ axis is parallel to the cylinders axis), and the $y$ axis being the depth (as shown in Fig. 1), it is also possible to calculate the stresses behavior in function of the axes.

According to Norton [1] and Johnson [4], the maximum pressure $P_{o}$ is found at the center of the contact region, where $y=0$, and its value is obtained through Eq. (1)

$P_{0}=\frac{2 \cdot F}{\pi \cdot a \cdot l}$

According to Norton [1], the width $2 a$ from the contact rectangle depends on the applied load $F$, geometrical and mechanical properties, and is given by Eq. (2): 
$a=\sqrt{\left(\frac{2}{\pi}\right) \cdot\left(\frac{\frac{1-v_{1}^{2}}{E_{1}}+\frac{1-v_{2}^{2}}{E_{2}}}{\frac{1}{2} \cdot\left(\frac{1}{R 1}+\frac{1}{R 2}\right)}\right) \cdot\left(\frac{F}{L}\right)}$

Inside the contact rectangle, there is a stress field whose maximum value is found at the centerline, and it decreases as it moves away from the center until it becomes zero at the border, being null outside the contact region [3].

The normal and shear stresses behavior along the axis where the load is applied (coincident to the $y$ axis) is described using Eqs. (3)-(5), and the principal shear stress is given by Eq. (6) [4].

$$
\begin{aligned}
& \sigma_{x}=-\frac{P_{0}}{a} \cdot\left\{\frac{\left(a^{2}+2 y^{2}\right)}{\sqrt{\left(a^{2}+y^{2}\right)}}-2 y\right\} \\
& \sigma_{z}=-2 \cdot P_{0} \cdot v \cdot\left\{\sqrt{\left(1+\frac{y^{2}}{a^{2}}\right)}+\frac{y}{a}\right\} \\
& \sigma_{y}=P_{0} \cdot a \cdot\left(\frac{1}{\sqrt{\left(a^{2}+y^{2}\right)}}\right)
\end{aligned}
$$

Fig. 2 Examples of rails and wheels found in the industry [22]
$\tau_{1}=-\frac{P_{0}}{a} \cdot\left\{y-\left(\frac{y^{2}}{\sqrt{\left(a^{2}+y^{2}\right)}}\right)\right\}$

The von Mises equation is used for a better understanding of the stresses acting simultaneously, as described in Eq. (7) $[1,3,21]$.

$\sigma^{\prime}=\sqrt{\frac{\left(\sigma_{x}-\sigma_{y}\right)^{2}+\left(\sigma_{y}-\sigma_{z}\right)^{2}+\left(\sigma_{x}-\sigma_{z}\right)^{2}+6 \cdot\left(\tau_{1}\right)^{2}}{2}}$

The stresses in the contact area are distributed elliptically. The behavior of each one is described according to Eqs. (8) and (9) [4].

$\sigma_{x}=\sigma_{z}=-P_{0} \cdot \sqrt{\left(1-\frac{x^{2}}{a^{2}}\right)}$

$\sigma_{y}=-2 \cdot v \cdot P_{0} \cdot \sqrt{\left(1-\frac{x^{2}}{a^{2}}\right)}$

\section{Materials and methods}

\subsection{Analytical approach}

The analytical results were obtained based on a real scenario where a wheel is in contact with a rail, both made of structural steel. Figure 2 shows two examples of rail
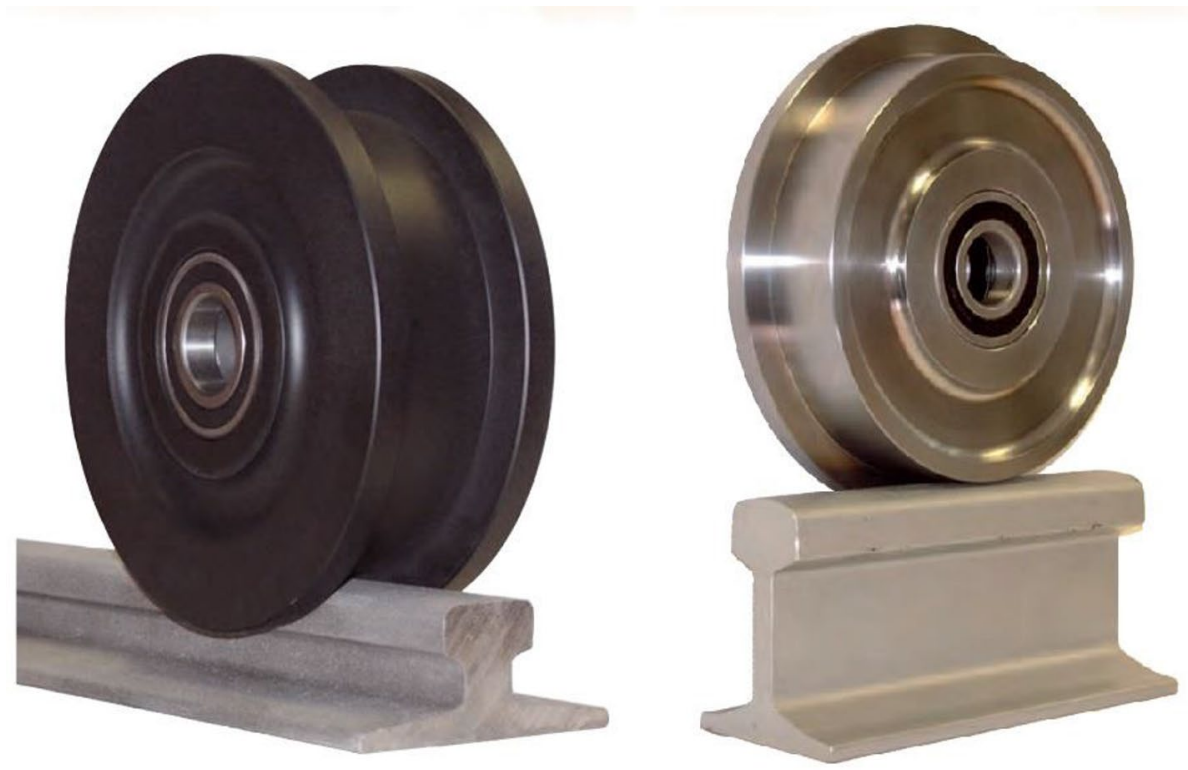
and wheel found in the market. Analyzing the contact between the bodies, it can be deduced that there is a cylinder (wheel) and a flat plate (rail) contact. In order to adapt the Hertz equations for two cylinders with parallel axes for this situation, the radius of the second body must tend to infinity $\left(R_{2} \rightarrow \infty\right)$.

All data used in the calculations were based on real application from Norton [1], and they are shown in Table 1.

\subsection{Numerical approach}

The contact between a wheel and a rail can be simplified to $1 / 2$ of the model due to the contact symmetry, maintaining the same characteristics of the whole model [17, 23]. Therefore, a computational $1 / 2$ model using the FEM software Ansys of the cylinder and the flat plate was constructed, as seen in Fig. 3.

\subsubsection{Constraints}

In order to simulate the symmetry of the complete model, the lower side of the flat plate was fixed with constraints in all directions, while the side faces were allowed to move only on the vertical direction ( $y$ axis). Therefore, the simplified model behaves exactly as its original version.

\subsubsection{Loads}

A compressive load of $22,241.11 \mathrm{~N}$ was applied on the upper side of the cylinder, on the negative direction of the $y$ axis.

\subsubsection{Mesh}

The mesh was at first generated automatically by the software. But, this first mesh was not accurate because Ansys generated an equal distribution of the elements on both bodies. In order to optimize the results, it was necessary to refine the mesh in all critical parts to the behavior of the elements, which is the point where both surfaces touch each other. After the refinement of the mesh, the results showed an improvement and got more consistent with the expected results. The software used in this simulation has three types of mesh: hexagonal (hex), tetrahedral and

Table 1 Values of the parameters used [1]

\begin{tabular}{ll}
\hline Wheel diameter $D$ & $301.80 \mathrm{~mm}(12 \mathrm{in})$. \\
Wheel length I & $22.23 \mathrm{~mm}(0.875 \mathrm{in})$. \\
Applied load $F$ & $22,241.11 \mathrm{~N}(5000 \mathrm{lb})$ \\
$E$ (Structural steel) & $200.00 \mathrm{GPa}$ \\
$u$ (Structural steel) & 0.28 \\
\hline
\end{tabular}

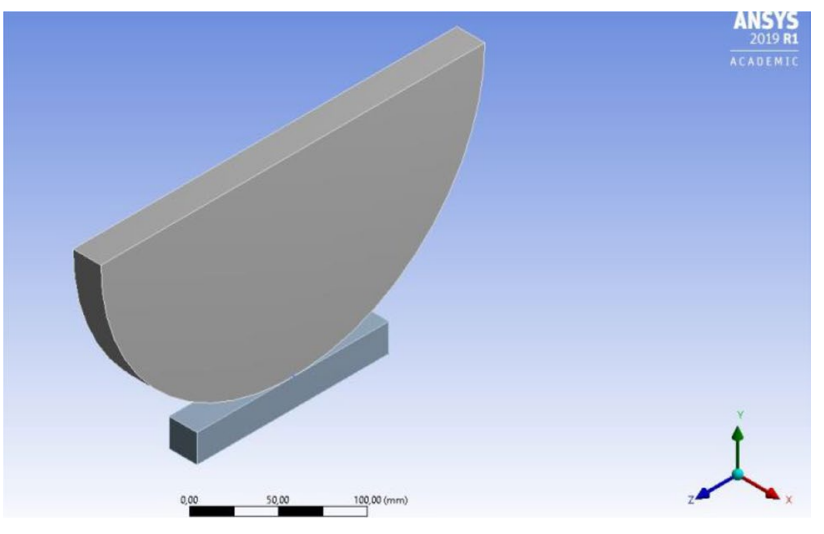

Fig. 3 Model of the cylinder and flat plate

polyhedral. The hex type had the best results when compared with the others, so the results seen in this article was using the first type of mesh.

The final mesh has 27,968 nodes and 15,551 elements. It is important to highlight that the Academic License of Ansys was used, and this version is limited to 32,000 nodes. This limitation has proven to be a significant factor on the quality of the mesh, because if there was an unlimited capacity of mesh, the results are expected to be even better. Thus, the mesh generated was the optimal with the offered tools.

\subsubsection{Surface contacts}

The contact of the external surface of the cylinder and the upper side of the flat plate was modeled using "Frictionless-Solid to Solid"type. Hence, it is defined that the bodies don't have any friction between its surfaces, respecting the third assumption from the Hertz theory.

\section{Results}

The results obtained from the analytical (using Hertz equations) and numerical approach (using the FEM simulation) are displayed in Table 2, where it is also presented

Table 2 Analytical and numerical results

\begin{tabular}{lccc}
\hline & Analytical (MPa) & Numerical (MPa) & $\begin{array}{l}\text { Percentage } \\
\text { deviation (\%) }\end{array}$ \\
\hline$\sigma_{x}$ & -253.69 & -238.41 & 6.03 \\
$\sigma_{y}$ & -453.08 & -542.63 & 19.78 \\
$\sigma_{z}$ & -453.08 & -429.84 & 5.12 \\
$\tau_{y z}$ & 135.90 & 129.84 & 4.46 \\
$P_{0}$ & 484.31 & 487.21 & 0.60 \\
\hline
\end{tabular}


a percentage deviation between the results, in order to show the percentage difference from the stresses obtained by both approaches.

From the values from Table 1, the results for $a, B, P_{0}$, as well as the stresses behavior at the contact plane between the bodies and along the $y$ axis are calculated. Through Eqs. (1) and (2), it was obtained that $P_{0}=484.31 \mathrm{MPa}$ and $a=1.31 \mathrm{~mm}$. This maximum pressure $P_{0}$ is found at the centerline of the contact rectangle between the cylinder and the flat plate. The rectangle width is $2 a$, and outside this region all stresses are null.

\subsection{Analytical results}

\subsubsection{Stresses along the $y$ axis}

Figure 4 illustrates the behavior of the stresses $\sigma_{x^{\prime}} \sigma_{y^{\prime}} \sigma_{z^{\prime}} \tau_{1}$ and the von Mises stress $\sigma^{\prime}$ along the axis of the applied load, i.e., the $y$ axis. All results from Fig. 4 were calculated through Eqs. (3)-(7).

In Fig. 4 it is visible that all three normal stresses are compressive and negative. They have maximum values when $y=0$ and tend to drop quickly as the depth $y$ increases. The stresses $\sigma_{x}, \sigma_{y}$ and $\sigma_{z}$ at $y=0$ are principal due to the lack of shear stresses at this point. The triaxial stress field is caused by the Poisson's ratio, because when a body is compressed against another in the $y$ direction, the vicinity tends to compress itself in order to avoid these deformations [18].

The maximum shear stress is positive and is found under the contact surface between the bodies, at a depth of $0.78 a$, and its maximum value reaches around $0.30 P_{0}$. This happens due to the combination of all stresses in the Mohr's circle [18].

The von Mises stress represents the single uniaxial stress that causes the same effect as all stresses combined. Its peak is found at a depth of $0.73 a$, and it is highly influenced by the shear stress, as both of them have a similar curve, as seen in Fig. 4. Therefore, it is possible to conclude that the shear stress is the most critical for the appearance of cracks that can cause failures and malfunctions.

\subsubsection{Stresses on the plane $x z$}

Figure 5 illustrates how $\sigma_{x^{\prime}} \sigma_{y}$ and $\sigma_{z}$ behave on the contact plane between the two bodies. All results were acquired using Eqs. (8) and (9).

The three stresses in Fig. 5 have their maximum values at the center of the contact, and they decrease elliptically until reaching the borders, when they became null. The stresses are found only in the contact rectangle, i.e., outside this area, where $x>a$ and $x<-a$, the Hertz stress are non-existent.

At the contact region $(-a<x<a)$ the stresses are distributed elliptically, varying from zero until its maximum at the center, and then they decrease again until zero.

\subsection{Numerical results}

Figure 6 shows the absolute stresses obtained solving the simulation proposed on item 3.2. The critical region is found at the centerline of the contact region between the cylinder and the flat plate. All normal stresses are maximum at the contact plane where $y=0$, and they tend to decrease quickly as the depth is increased.

In addition to the stress analysis, it was also studied the deformation area caused by the contact from both surfaces. Figure 7 represents the contact surface between the two elements ( $x z$ plane), and it is possible to see that

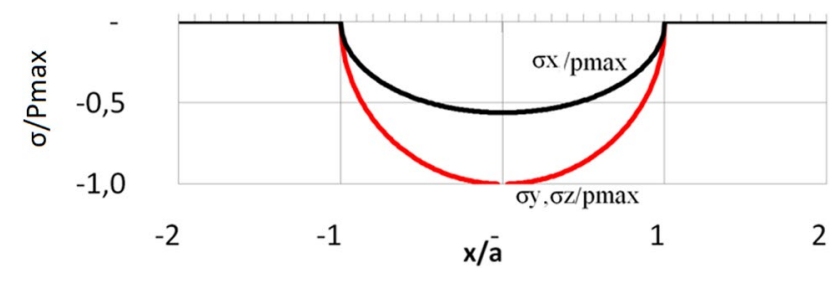

Fig. 5 Stresses on the contact plane $x z$
Fig. 4 Stresses in the $y$ axis

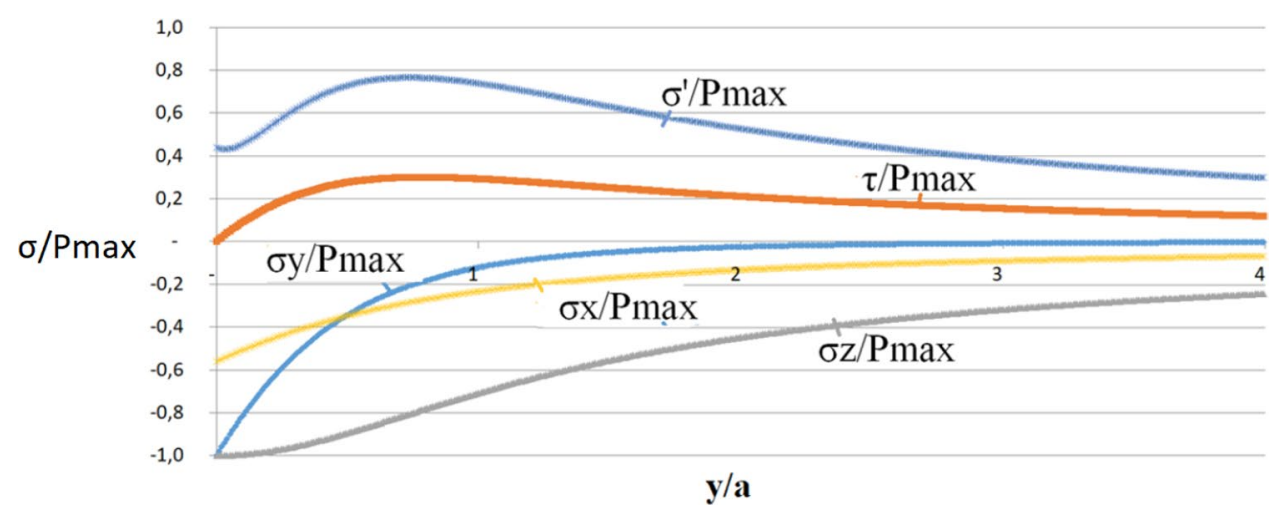

SN Applied Sciences 


A: Static Structural
Pressure
Type: Pressure
Unit: MPa
\begin{tabular}{|l|l}
\hline 453,08 Max \\
253,86 \\
150,77 \\
102,51 \\
10,53 \\
1,03 \\
0 Min
\end{tabular}
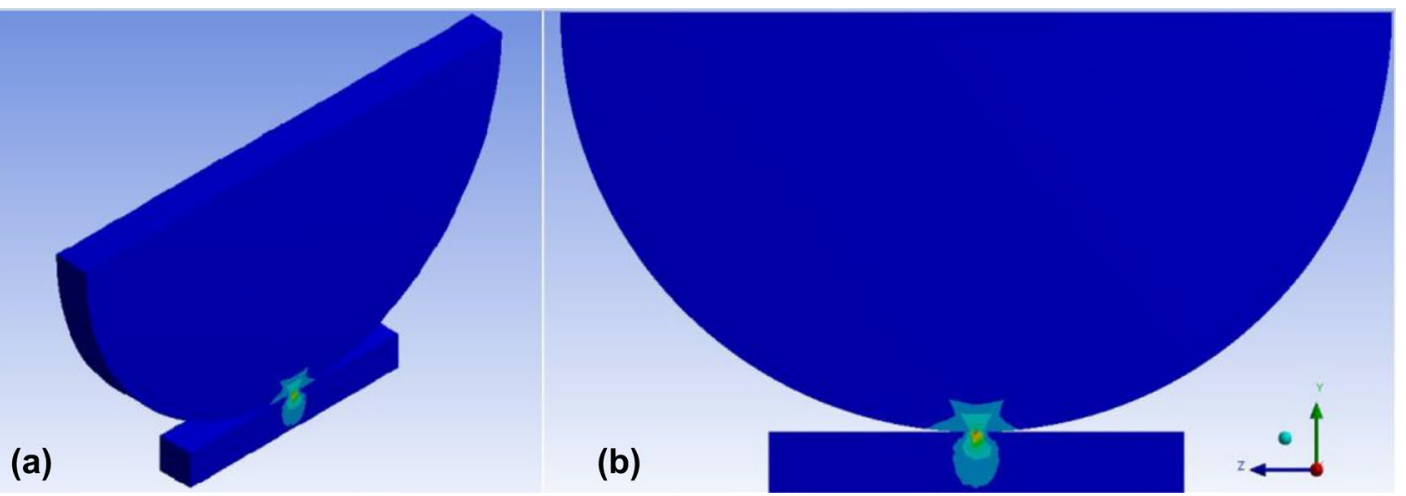

Fig. 6 Numerical results
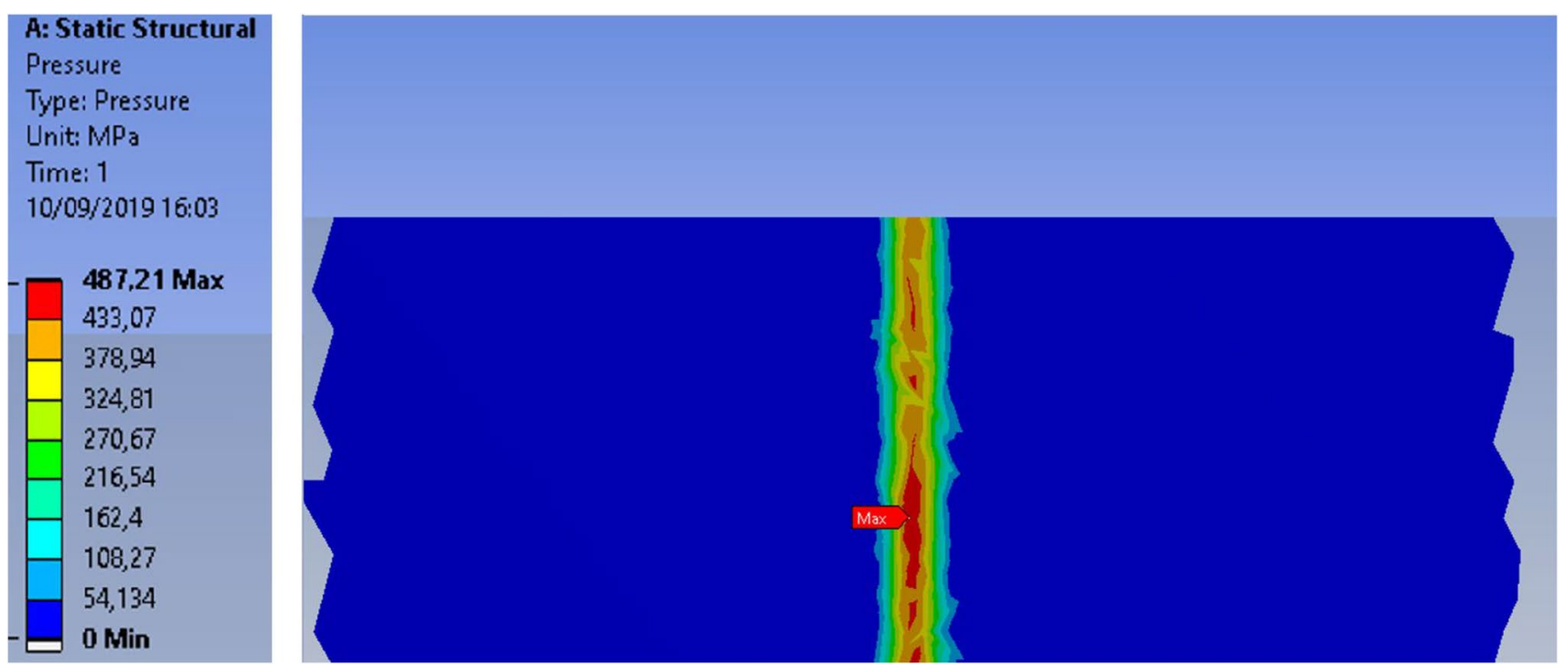

Fig. 7 Contact surface

the stresses are limited to a rectangle area and that the maximum pressure is found at its centerline. The stress decreases as it moves away from the centerline, until reaches zero at the border. The area affected by the stress from the numerical simulation is $66.32 \mathrm{~mm}^{2}$, while the area calculated by the analytical equations is $58.24 \mathrm{~mm}^{2}$, thus the percentage deviation from both results is around $13.87 \%$.

\section{Discussion}

Comparing the results obtained from the numerical and analytical approach, it is seen that all results are significantly close, with a mean percentage deviation of $7.20 \%$. The only major discrepancy was found in $\sigma_{y}$, where the numerical value obtained was relatively far from the analytical value (19.78\%). This inconsistency can be justified by the inaccuracy from the Hertz equations regarding to the thickness of the bodies, that is, the Eqs. (3)-(6) do not consider the thickness of the flat plate, therefore some of the results between the FEM simulation and the analytical values can be different regarding the axis in the same direction as the depth.

The theory behind the Hertz contact stresses seen different works $[1,4,18]$ says that the maximum pressure and shear stresses are the most critical ones regarding the appearance of cracks that might cause the failure by RCF on the element. Considering that the percentage deviation from both stresses is around $2.53 \%$, the model can be validated for this simulation. It is possible to conclude that the model is in good terms with the reality and it can be used to predict the behavior in real scenarios where the contact stresses have a great significance.

\section{SN Applied Sciences}




\section{Conclusions}

In this paper, an analytical approach was proposed to study the contact between a cylinder and a flat plate (representing a rail and a wheel), using the Hertz theory for contact surfaces between two cylinders (with one of the radius tending to infinity), and with that it was expected to create a model that represents faithfully the real behavior of the elements. It was also proposed a numerical method through a FEM model using the software Ansys. The results showed that all normal stresses are maximum on the contact plane and tend to zero quickly as the depth increases, while the shear stress has its peak at a depth of $0.78 a$, and the von Mises stress is higher when $y=0.73 a$.

On the contact zone between the bodies, the stress is distributed elliptically, and the peak is exactly at the center of the contact, decreasing until zero when reaches the borders. Outside the rectangular contact zone the Hertz stress is always null.

There is a similarity between the behavior of the shear stress and the von Mises stress, so it is possible to conclude that the shear stress is the most significant for the appearance of cracks, since the von Mises shows how all normal and shear stress act together simultaneously.

Comparing the data obtained from both approaches, the results were satisfactory, although the stress along the $y$ axis obtained with the FEM software is a little far from the analytical result, with a percentage deviation of $19.78 \%$. This discrepancy occurred due to the mesh limitations from the software used for the simulations. In spite of this disparity, the model was validated because the results for the most important stresses for the appearance of cracks, i.e., the shear stress and the maximum pressure, are significantly close between both approaches. With that, it was concluded that the simulations are in good terms with reality, so this work can be used as a basis for future contact stresses studies, as well as to predict real scenarios.

Acknowledgements This study was financed in part by the Coordenação de Aperfeiçoamento de Pessoal de Nível Superior, Brasil (CAPES), Finance Code 001 and CNPq.

\section{Compliance with ethical standards}

Conflict of interest The authors declare that they have no conflict of interest.

Human and animal rights statement This article does not contain any studies involving human participants or animals performed by any of the authors.

\section{References}

1. Norton RL (2013) Projeto de máquinas. Bookman, Brazil

2. Arakere NK (2016) Gigacycle rolling contact fatigue of bearing steels: a review. Int J Fatigue 93:238-249

3. Solanki MT, Vakharia D (2017) A finite element analysis of an elastic contact between a layered cylindrical hollow roller and flat contact. Ind Lubr Tribol 69(1):30-41

4. Johnson KL (1987) Contact mechanics. Cambridge University Press, Cambridge

5. Deng S, Hua L, Han X, Huang S (2014) Investigation of rolling contact fatigue cracks in ball bearings. Int J Fract 188(1):71-78

6. Deng S, Hua L, Han X, Huang S (2013) Finite element analysis of fatigue life for deep groove ball bearing. Proc Inst Mech Eng Part L J Mater Des Appl 227(1):70-81

7. Solanki MT, Vakharia D (2017) Extending hertz equation for an elastic contact between a layered cylindrical hollow roller and flat plate through an experimental technique. Ind Lubr Tribol 69(2):312-324

8. Li F, Hu W, Meng Q, Zhan Z, Shen F (2018) A new damagemechanics-based model for rolling contact fatigue analysis of cylindrical roller bearing. Tribol Int 120:105-114

9. Mohd Yusof NF, Ripin ZM (2014) Analysis of surface parameters and vibration of roller bearing. Tribol Trans 57(4):715-729

10. Hao Y, Shi Y (2015) Contact stress analysis for a pair of aluminum matrix composite helical gear and steel worm. J Reinf Plast Compos 34(3):213-221

11. Hwang SC, Lee JH, Lee DH, Han SH, Lee KH (2013) Contact stress analysis for a pair of mating gears. Math Comput Model 57(1-2):40-49

12. Patil SS, Karuppanan S, Atanasovska I, Wahab AA (2014) Contact stress analysis of helical gear pairs, including frictional coefficients. Int J Mech Sci 85:205-211

13. Gonzalez-Perez I, Iserte JL, Fuentes A (2011) Implementation of Hertz theory and validation of a finite element model for stress analysis of gear drives with localized bearing contact. Mech Mach Theory 46(6):765-783

14. Nejad RM, Shariati M, Farhangdoost K (2016) Effect of wear on rolling contact fatigue crack growth in rails. Tribol Int 94:118-125

15. Xiao JH, Zhang FC, Qian LH (2014) Contact stress and residual stress in the nose rail of a high manganese steel crossing due to wheel contact loading. Fatigue Fract Eng Mater Struct 37(2):219-226

16. Jaifu A, Raeon S, Pimsarn M (2018) Study of fatigue crack initiation location of wheel and rail under rolling contact using finite element method. In: MATEC web of conferences, vol 192. EDP Sciences, p 02012

17. Subhash G, Arakere N, Bhattacharyya A, Londhe N (2017) A new approach towards life prediction of case hardened bearing steels subjected to rolling contact fatigue. Mater Perform Charact 6(4):656-677

18. Juvinall RC, Marshek KM (2008) Projeto de Componentes de Máquinas. Grupo Gen-LTC

19. Deng S, Qin X, Huang S (2015) A study on the effect of subsurface crack propagation on rolling contact fatigue in a bearing ring. J Mech Sci Technol 29(3):1029-1038

20. Hongyan C, Ran A, Ligang C (2016) Applicability of the Hertz contact theory to rolling rubber cylinders model. Int J Mech Eng Appl 4(6):242-248

21. Brezeanu LC (2013) Contact stresses between two cylindrical bodies with parallel axes: analysis by FEM. Scientific Bulletin of the "Petru Maior" University of Targu Mures 10(2):9 
22. Brauer (2019) Wheels and Castors Catalog. Brauer Ltd. http:// www.brauer.co.uk/files/downloads/23_wheels\%20catalogu e\%20-\%20english.pdf

23. Satyanarayana S, Melkote SN (2004) Finite element modeling of fixture-workpiece contacts: single contact modeling and experimental verification. Int J Mach Tools Manuf 44(9):903-913
Publisher's Note Springer Nature remains neutral with regard to jurisdictional claims in published maps and institutional affiliations. 